\title{
Properties of Greedy Geographical Routing in Spontaneous Wireless Mesh Networks
}

\author{
Schiller, Eryk ; Starzetz, Paul ; Theoleyre, Fabrice ; Duda, Andrzej
}

\begin{abstract}
We analyze greedy geographical routing in spontaneous wireless mesh networks to show several interesting properties. First, we can approximate the dependence of packet loss probability on the mean node rank with a Fermi-Dirac function. When the mesh network grows, it becomes opaque to packets regardless of the average node rank. We also show that packet loss probability in mesh networks with greedy geographical routing does not exhibit the behavior of percolating systems. Finally, we propose an analytical model of greedy geographical routing and use it to derive packet loss probability.
\end{abstract}

DOI: https://doi.org/10.1109/GLOCOM.2007.937

Posted at the Zurich Open Repository and Archive, University of Zurich

ZORA URL: https://doi.org/10.5167/uzh-174654

Conference or Workshop Item

Accepted Version

Originally published at:

Schiller, Eryk; Starzetz, Paul; Theoleyre, Fabrice; Duda, Andrzej (2007). Properties of Greedy Geographical Routing in Spontaneous Wireless Mesh Networks. In: IEEE GLOBECOM 2007-2007 IEEE Global Telecommunications Conference, Washington, DC, 26 December 2007 - 30 December 2007. IEEE, 4941-4945.

DOI: https://doi.org/10.1109/GLOCOM.2007.937 


\title{
Properties of Greedy Geographical Routing in Spontaneous Wireless Mesh Networks
}

\author{
Eryk Schiller, Paul Starzetz, Fabrice Theoleyre, Andrzej Duda \\ LIG - Grenoble Informatics Laboratory* \\ Grenoble, France \\ Email: \{schiller, starzetz, theoleyr, duda\}@imag.fr
}

\begin{abstract}
We analyze greedy geographical routing in spontaneous wireless mesh networks to show several interesting properties. First, we can approximate the dependence of packet loss probability on the mean node rank with a Fermi-Dirac function. When the mesh network grows, it becomes opaque to packets regardless of the average node rank. We also show that packet loss probability in mesh networks with greedy geographical routing does not exhibit the behavior of percolating systems. Finally, we propose an analytical model of greedy geographical routing and use it to derive packet loss probability.
\end{abstract}

\section{INTRODUCTION}

We consider wireless mesh networks composed of a large number of mesh routers providing connectivity to mesh client stations. We assume that mesh routers may only move occasionally, whereas client terminals are potentially highly mobile. Neighbor mesh routers that are within a transmission range directly communicate over wireless links. End-to-end connectivity between any source and destination in the mesh requires multi-hop packet forwarding by intermediate nodes. We focus on spontaneous wireless mesh networks that begin to appear in highly populated areas to provide cheap network connectivity to a community of end users. One of their characteristics is self-forming-users just add another mesh router in some place to increase the coverage.

Unlike traditional approaches, geographical routing presents interesting properties for spontaneous wireless mesh networks: it does not require any information on the global topology, but uses the position of the destination to forward packets. Several variants of geographical routing have been proposed, the most familiar one being greedy forwarding in which a node forwards a packet to the neighbor that has the least distance to the destination. Greedy forwarding guarantees loop-free operation, but packets may be dropped at concave nodes that have only neighbors in the backward direction. Geographical routing only requires local routing tables restricted to the closed neighborhood resulting in a low overhead of their creation and maintenance. However, it relies on the information about the physical location of nodes, which can be provided by means of a global positioning techniques such as GPS. In the absence

${ }^{*}$ LIG is a joint research laboratory of CNRS (Centre National de la Recherche Scientifique), INRIA (Institut National de Recherche en Informatique et Automatique), INP Grenoble (Institut Polytechnique de Grenoble), UJF (Université Joseph Fourier), and UPMF (Université Pierre-Mendès-France). of GPS, for instance indoors, the location information can be obtained from relative positioning based on estimation of the signal strength [1].

In this paper, we study general properties of geographical routing in spontaneous wireless mesh networks. We use a circular arena to place wireless nodes and study routing performance for different mesh sizes and densities. We adopt the Unit Disk Graph (UDG), a common connectivity model for representing multi-hop wireless networks. We consider it as a kind of a first-order approximation useful for studying main trends in performance behavior. Our goal is to identify and understand the behavior of greedy geographical routing and to quantify its performance.

We start with the definition and description of a graph model based on a uniform distribution of nodes in a circular arena. We derive the distribution of the node rank (the number of its neighbors, also called node degree). Then, we analyze greedy geographical routing and show how packet loss probability varies in function of the network size. In particular, we demonstrate that the probability of packet delivery (probability of reaching the destination) is not a percolation phenomenon in contrast to network connectivity related to the existence of a network path between any two nodes. We also propose an analytical model of greedy geographical routing and use it to derive probability of packet loss. Our simulation results come from a dedicated graphical simulator written in $\mathrm{C}++$ able to handle up to a million nodes.

\section{RELATED WORK}

Several authors have addressed the problem of greedy geographical routing in wireless mesh or ad hoc networks. Bose et al. have done a pioneering work on greedy geographical routing in which a node chooses to forward a packet to the neighbor closest to the destination [2]. To cope with concave nodes, the authors propose a method called face-routing to surround the hole. However, face-routing requires a planar graph, i.e. there is no crossing edges. For unit disk graphs, the construction of a Gabriel graph [3] or a relative neighborhood graph [4] leads to a planar graph without any connectivity loss. Kuhn et al. study asymptotically different geographical routing protocol and compare their performance [5]. Frey considers scalable geographical routing protocol and discuss recent improvements to greedy forwarding [6]. De et al. give 
the bounds on the hop distance in greedy routing [7]. In our work, we focus on the analysis of packet losses in greedy geographical routing due to a concave node on the route to the destination.

\section{Modeling SPONTANeOUS WIRELESS MESH NETWORKS}

\section{A. Unit disk graph}

We consider a spontaneous wireless mesh network with $N$ nodes uniformly distributed in a disk $\mathbb{D}$ of radius $L$ with area $A=\pi L^{2}$. The probability density function of node position $(x, y)$ is thus:

$$
p_{l}(x, y)= \begin{cases}\frac{1}{A} & (x, y) \in \mathbb{D} \\ 0 & (x, y) \notin \mathbb{D}\end{cases}
$$

We assume that nodes use omnidirectional antennas and their transmission range is $R$ with a perfect propagation model. Denoting by $d(\cdot, \cdot)$ the Euclidean distance between any two nodes, there is a link in the graph between nodes $n_{1}, n_{2}$, if $d\left(n_{1}, n_{2}\right)<R$.

\section{B. Properties of the graph}

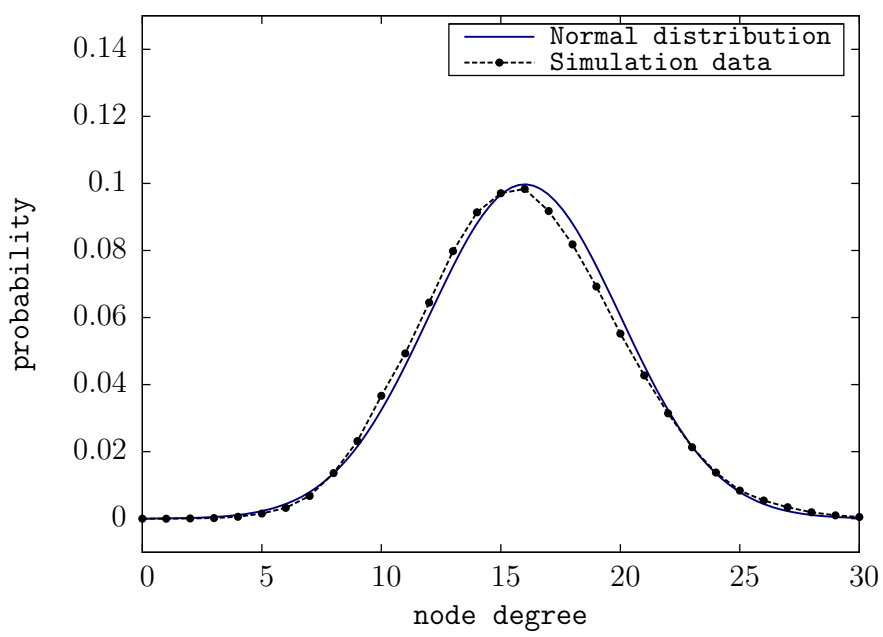

Figure 1. Probability distribution of $k, N=10000, L=1, R=0.04$.

First, we want to find the distribution of $k$, the rank of a node, i.e. the probability that a node has $k$ links with its neighbors. If we know $(x, y)$, the position of node $n_{1}$, the probability $p_{r}$ (resp. $q_{r}$ ) that node $n_{2}$ is inside (resp. outside) the disk of radius $R$ placed at $(x, y)$ is a Bernoulli distribution with parameters

$$
p_{r}=\frac{R^{2}}{L^{2}} \quad \text { and } \quad q_{r}=1-p_{r} .
$$

As the sum of Bernoulli distributions is a binomial distribution

$$
P_{k}=\left(\begin{array}{c}
N \\
k
\end{array}\right) p_{r}^{k} q_{r}^{N-k}
$$

the mean node rank $\bar{k}$ is $N p_{r}$ and its variance is $\operatorname{Var}[k]=$ $N p_{r} q_{r}$. For large values of $N$, the distribution of the node rank converges to the normal (Gaussian) distribution $\mathcal{N}$ :

$$
\lim _{N \rightarrow \infty} P_{k}=\mathcal{N}(\bar{k}, \operatorname{Var}[k]) \text {. }
$$

Figure 1 shows a good match between the above theoretical result and simulation for $N=10000$ nodes and $L=1, R=$ 0.04. In this model, $\bar{k}$ and $\operatorname{Var}[k]$ are finite, because from the relation

$$
p_{r}=\frac{\bar{k}}{N}=\frac{R^{2}}{L^{2}}
$$

we can use $R=L \sqrt{\frac{\bar{k}}{N}}$ to find its value for a network with a given mean node rank $\bar{k}$. Likewise, in the simulations below, we use the relation $\bar{k}=N \frac{R^{2}}{L^{2}}$ to generate graphs with different values of the mean node rank by varying $N, R$, and $L$.

\section{GREEDY GEOGRAPHICAL ROUTING}

In this section, we study the behavior of greedy geographical routing in a spontaneous mesh network: nodes forward packets based on the Euclidean distance $d(\cdot, \cdot)$ by choosing the next hop with the least distance to the destination. If there is no such neighbor, the packet is dropped. We assume that all nodes know the positions of their neighbors.

We start with simulations of a large number of unit disk graphs for a given mean node rank $\bar{k}$ and observe packet loss probability $p_{e}$ of greedy geographical routing for a sufficiently large sequence of randomly chosen source and destination nodes (we simulate transmission of over $10^{6}$ packets between randomly chosen nodes). We then derive an experimental expression for packet loss distribution as a function of the mean node rank $\bar{k}$.

\section{A. Packet loss probability for the varying mean node rank}

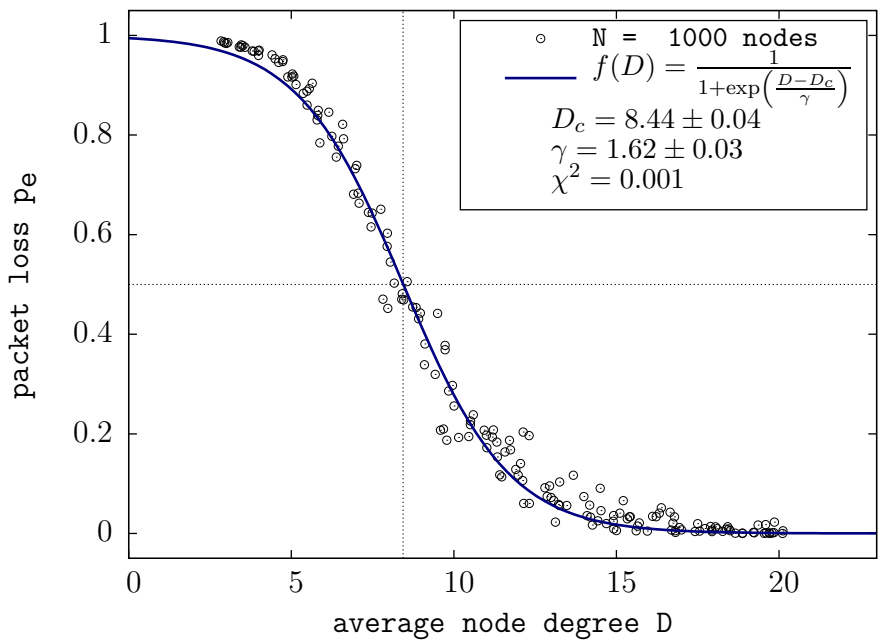

Figure 2. Packet loss probability for $N=1000$ nodes, $\bar{k}_{0}=8.44 \pm$ $0.04, \gamma=1.62 \pm 0.03$.

Figure 2 presents example simulation results, the experimental distribution of packet loss probability $p_{e}$ in function of the mean node rank $k$ for $N=1000$. A point in the figure represents packet loss probability $p_{e}$ obtained in a simulation of one randomly generated graph of a given mean node rank $\bar{k}$. We have confirmed by $\chi^{2}$ testing that the distribution can 
be approximated by a Fermi-Dirac function described by the equation:

$$
p_{e}(\bar{k})=\frac{1}{1+\exp \left(\frac{\bar{k}-\bar{k}_{0}}{\gamma}\right)}
$$

We have used the function to find the value of the critical average node rank $\bar{k}_{0}$ for which packet loss probability equals $p_{e}\left(\bar{k}_{0}\right)=0.5$ by fitting $\bar{k}_{0}$ and $\gamma$ to simulation results with the least-square method. We can see in Figure 2 a steep decrease of packet loss probability $p_{e}(\bar{k})$ around the critical average node rank of $\bar{k}_{0}=8.44$. For $N=4000$, the results are similar, but astonishingly the critical average node rank $\bar{k}_{0}$ does not stay constant, but rather slowly grows with the increasing mesh size, i.e. $\bar{k}_{0}=9.90$ in this case.

Our observation is that packet losses occur only in fairly few places in the simulated graphs. For example in a graph with $N=1000$ nodes and for $p_{e}=0.5$, there are just few nodes (typically 10-15) that drop packets. These nodes can be identified as topology defects called concave nodes, because they only have neighbors in the backward directionthey appear at the border of holes in the mesh corresponding to nodes surrounding an empty concave region without links. Thus, an adequate model of the interaction of the geometry of the unit disk graph with greedy routing is a forest of defects with a constant (for a given $\bar{k}_{0}$ ) defect probability per unit area of the mesh. This means that a unit area of the graph has a specific opacity for packets under greedy routing analogous to the light passing through an opaque medium (extinction).

Let us study the behavior of the critical average node rank $\bar{k}_{0}$ and packet loss probability when the size of the network increases. Let $p_{e}^{(1)}=\epsilon$ and $p_{t}^{(1)}=1-\epsilon$ denote packet loss and transmission probabilities per unit area, respectively. If we quadruple the area of the mesh network while keeping the average node rank $\bar{k}$, the average distance a packet travels in the quadrupled mesh doubles. Thus, a packet will cross two units of the graph on the average and the transmission probability $p_{t}^{(2)}$ in the quadrupled mesh becomes $p_{t}^{(2)}=(1-$ $\epsilon)^{2}$. So, packet loss probability in the quadrupled mesh will be $p_{e}^{(2)}=1-(1-\epsilon)^{2}=1-\left(1-p_{e}^{(1)}\right)^{2}$, since the packet can be lost on each half of the way it travels (losses are approximately independent, if we neglect the interaction of the borders).

We can apply the above result to predict the value of the critical average node rank $\bar{k}_{0}$ for the 4000-node simulation using Eq. 6 and the value of the critical rank from the 1000node simulation:

$$
0.5=1-\left(1-p_{e}^{(1)}\right)^{2}=1-\left(1-\frac{1}{1+\exp \left(\frac{\bar{k}-\bar{k}_{0}}{\gamma}\right)}\right)^{2}
$$

, which defines a new critical value of $\bar{k}$ for which $p_{e}^{(2)}=0.5$. Putting the values $\bar{k}_{0}=8.44, \gamma=1.62$ obtained for 1000 nodes into Eq. 7 and solving for $\bar{k}$ yields the value of $\bar{k}_{0} \approx$ 9.87 that fits the value of $\bar{k}_{0}=9.90$ from the simulation of 4000 nodes quite well. This enables us to estimate $\overline{k_{0}}$ of a larger network by simulating a much smaller mesh network.

We can further derive an approximate expression for packet loss probability in a mesh with the area of $4^{m}$ units by generalizing the above reasoning. We obtain an expression for the critical node rank $\bar{k}_{0}(m)$ given that the critical node rank $\bar{k}_{0}$ for the unit mesh is known: $p_{e}^{(m)}=1-\left(1-p_{e}^{(1)}\right)^{m}$. After inserting it into Eq. 6 and solving for $\bar{k}(m)$ we get:

$$
\lim _{m \rightarrow \infty} \bar{k}_{0}(m)=\infty \quad \text { and } \quad \lim _{m \rightarrow \infty} p_{e}^{(m)}=1 .
$$

Thus, an infinite mesh network under greedy geographical routing is completely opaque independently of the average node rank. This also means that the performance of greedy forwarding becomes worse with increasing mesh size when the mean node rank is kept constant. We have compared our

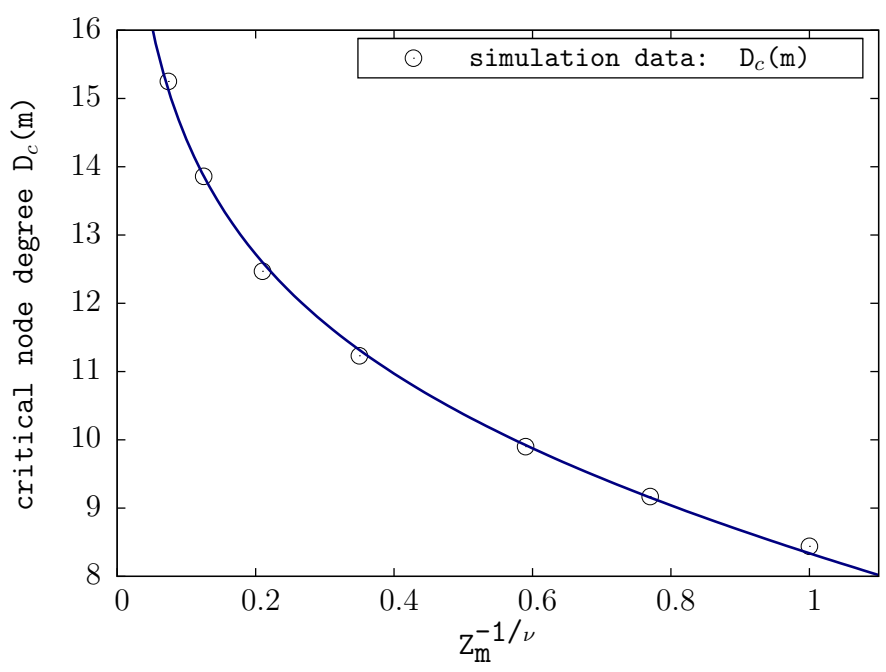

Figure 3. Packet loss probability in mesh networks with greedy geographical routing in mesh networks does not behave like a percolating system.

results on greedy geographical routing in spontaneous mesh networks with the classical percolation theory [8]. It states that an infinite percolating system needs to have a unique but finite critical average node rank $\bar{k}_{0}$ for which a measurable quantity of interest (in our case packet loss probability) experiences a sharp transition. The critical average node rank may slightly depend on the size of the observed system (so called a finite size effect), but it can be estimated for an infinite system size, if a sequence of critical average node ranks $\bar{k}_{0}(m)$ for differently-sized finite systems is plotted against $Z_{m}^{-\frac{1}{\nu}}$, that is the system size $Z_{m}$ (corresponding to the radius $L$ of the mesh network in our case) to the power of a dimension dependent, but universal percolation exponent $-\frac{1}{\nu}$. The exponent is known to be constant and equal to $\nu=\frac{4}{3}$ for any two dimensional system. The crossing of the resulting line with the $x$-axis should then give the critical average node rank for an infinite system.

Figure 3 presents the corresponding percolation graph. We can see that no straight line can be drawn in the case of greedy geographical routing in a mesh network, but rather we can observe an exponential growth of the critical average node rank. Thus, despite the fact that a mesh network itself is a percolating system in the sense of the global connectivity [9], [10], this property does not hold for greedy geographical routing in spontaneous mesh networks with packet loss probability 
as the percolation measure.

\section{Analytical Model of Greedy Geographical ROUTING}

In this section, we present an analytical model for packet loss probability $p_{e}$ (and thus for the successful transmission probability $p_{t}$ of delivering a packet) in a mesh network with greedy geographical routing and a given average node rank for any randomly chosen source and a destination.

\section{A. Probability that a neighbor is closer to the destination}

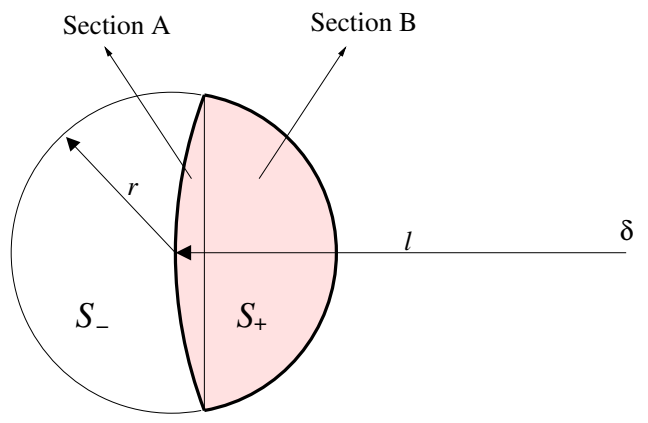

Figure 4. Packet loss model

Let us consider two disks $\mathbb{D}(0, R)$ and $\mathbb{D}(D, l)$ where $|D-l|<R$ (cf. Figure 4 for $l=D$ ). Disc $\mathbb{D}(0, R)$ is placed at the position of the node that forwards a packet, while $\mathbb{D}(D, l)$ is located at the destination node. For $l=D, S_{+}$(resp. $\left.S_{-}\right)$is the area of positions closer to (resp. farther of) the destination node. The intersection points $(x, y)$ of $\mathbb{D}(0, R)$ and $\mathbb{D}(D, D)$, $D \geq R$ satisfy the following system of equations:

$$
\begin{cases}x^{2}+y^{2} & =R^{2} \\ (x-D)^{2}+y^{2} & =D^{2}\end{cases}
$$

By solving it and summing up the area of disk sections $S_{+}=A+B$, we can express probability $p_{c}$ that a neighbor is closer to the destination as the ratio of $S_{+}$to the area of the smaller disk $\pi R^{2}$ :

$$
p_{c}=\frac{\left(R^{2} \arcsin \left(\xi_{R}\right)+D^{2}\left(\arcsin \left(\xi_{D}\right)-\xi_{D}\right)\right)}{\pi R^{2}},
$$

where

$$
\xi_{R}=\frac{1}{2 R} \sqrt{4 R^{2}-\frac{R^{4}}{D^{2}}}, \quad \xi_{D}=\frac{1}{2 D} \sqrt{4 R^{2}-\frac{R^{4}}{D^{2}}} .
$$

Figure 5 presents probability $p_{c}$ in function of the distance between the forwarding node and the destination expressed in multiples of range $R$. Its minimum value is $\left.p_{C}\right|_{D=R} \approx 0.39$ for the angle $\alpha$ corresponding to the case of $D=R$ :

$$
\alpha=2 \arcsin \left(\xi_{R}\right)=\frac{2}{3} \pi=120^{\circ}
$$

Note that $\lim _{D \rightarrow R^{+}} 2 \arcsin \left(\xi_{R}\right)=\alpha$, which means that if a node has a disk sector with an angle bigger then or equal $\alpha \geq 120^{\circ}$ not containing any neighbor, it may drop packets. Whether a packet is lost or not, depends on the direction towards the destination.

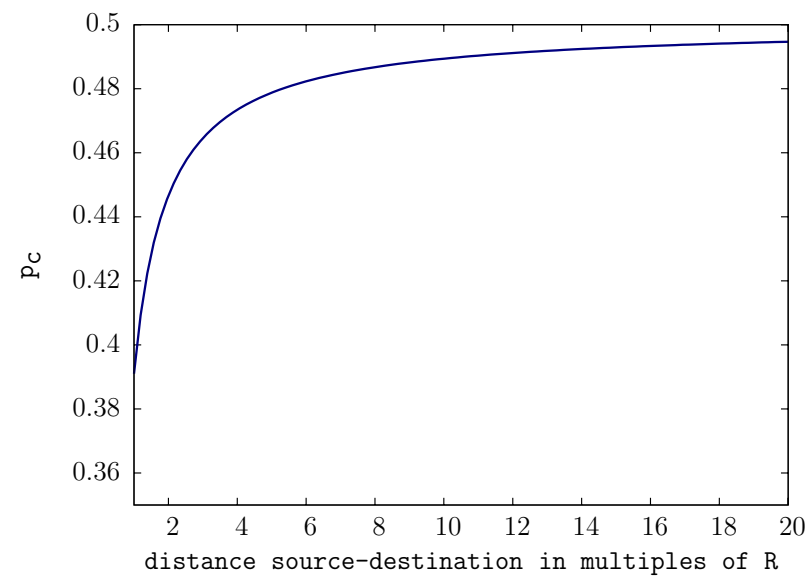

Figure 5. Probability $p_{c}$ that a neighbor is closer to the destination.

\section{B. Packet loss probability}

To find packet loss probability, we randomly choose source and destination nodes. Let denote the distance between them by $z$. We consider topology defects on the route to the destination-what is the probability of dropping a packet at a concave node. If not dropped, a packet will advance toward the destination by choosing the neighbor node for which the gain in distance is the best. So, first we compute the probability distribution of the distance that a packet will gain towards the destination when being forwarded by a given neighbor node. We then find the distribution of the maximum distance gain over the neighbors that are closer to the destination and we obtain the final result by considering the distribution of the distance between any two nodes in a mesh network.

Recall that $P_{k}$ given by Eqs. 2 and 3 is the probability that a given node has $k$ neighbors in its transmission range. Forwarding fails if packet arrives at a concave node (none of its neighbors is closer to the destination), which happens with probability $q_{c}=1-p_{c}$. We can express $p_{x}$, the probability of being a concave node as:

$$
p_{x}=\sum_{k=1}^{N}\left(\begin{array}{l}
N \\
k
\end{array}\right) p_{r}^{k} q_{c}^{k-1} q_{r}^{N-k}=\frac{\left(p_{r} q_{c}+q_{r}\right)^{N}-q_{r}^{N}}{q_{c}} .
$$

To find the probability distribution that a given neighbor is closer to the destination, we consider the case in Figure 4 for $D>R$ and $|D-l| \leq R$. To obtain the positions of the disk intersection, we can solve Eq. 9 for this case to obtain:

$$
\left\{\begin{array}{l}
y^{\prime}= \pm \sqrt{R^{2}-\frac{\left(-l^{2}+D^{2}+R^{2}\right)^{2}}{4 D^{2}}} \\
x^{\prime}=\frac{-l^{2}+D^{2}+R^{2}}{2 D}
\end{array}\right.
$$

Let us define

$$
\zeta=\frac{1}{l} \sqrt{R^{2}-\frac{\left(-l^{2}+D^{2}+R^{2}\right)^{2}}{4 D^{2}}} .
$$

The length of the arc of the larger circle located inside the smaller disc is $A_{L}=2 l \arcsin (\zeta)$. Knowing that

$$
S_{+}=\int_{l-R}^{l} A_{L} d l \rightarrow \int_{l-R}^{l} \frac{A_{L}}{S_{+}} d l=1,
$$


we can define the probability distribution that a given neighbor is closer to the destination by length $\delta$ as $p_{d}(\delta)=\frac{A_{L}}{S_{+}}$. When the forwarding node has on average $\bar{k}$ neighbors, approximately $k^{\prime}=p_{c} \cdot \bar{k}$ of them are closer to the destination. To find how far a packet advances towards the destination in one hop, consider neighbor $i, i=1, \ldots, k^{\prime}$. It is closer to the destination by length $\delta_{i}$ distributed according to $p_{d}$. Thus, the distance by which a packet advances towards the destination in one hop is $\Delta=\max \left\{\delta_{1} \ldots \delta_{k^{\prime}}\right\}$. We can find the distribution of $\Delta$ in the following way. First, we need to find the cumulative density function of $\delta_{i}$ as $F_{\delta_{i}}(x)=\int_{0}^{x} p_{d}(\delta) d \delta$. Then, the cumulative distribution of $\Delta$ is $F_{\Delta}(x)=\left(F_{\delta_{i}}(x)\right)^{k^{\prime}}$ and its probability density function $f_{\Delta}(x)=\frac{d}{d x} F_{\Delta}(x)$. So, if the destination is at distance $z$, a packet will reach the destination in approximately $\bar{h}=\frac{z}{E[\Delta]}$ hops on the average.

The probability density function of distance $z \in[0,2 L]$ between two randomly chosen points in a disc with radius $L$ is the following [11]:

$$
\Lambda(z)=\frac{z}{\pi L^{2}}\left(4 \arctan \frac{\sqrt{4 L^{2}-z^{2}}}{z}-\frac{z}{L} \sqrt{4-\left(\frac{z}{L}\right)^{2}}\right) .
$$

Finally, we can obtain the upper bound of packet loss probability as:

$$
p_{e}(\bar{k})=\int_{0}^{2 L} \Lambda(z)\left(1-\left(1-\left.p_{x}\right|_{D=R}\right)^{\left.\bar{h}\right|_{D=z}}\right) d z,
$$

where $R=L \sqrt{\frac{\bar{k}}{N}}$.

\section{Numerical results}

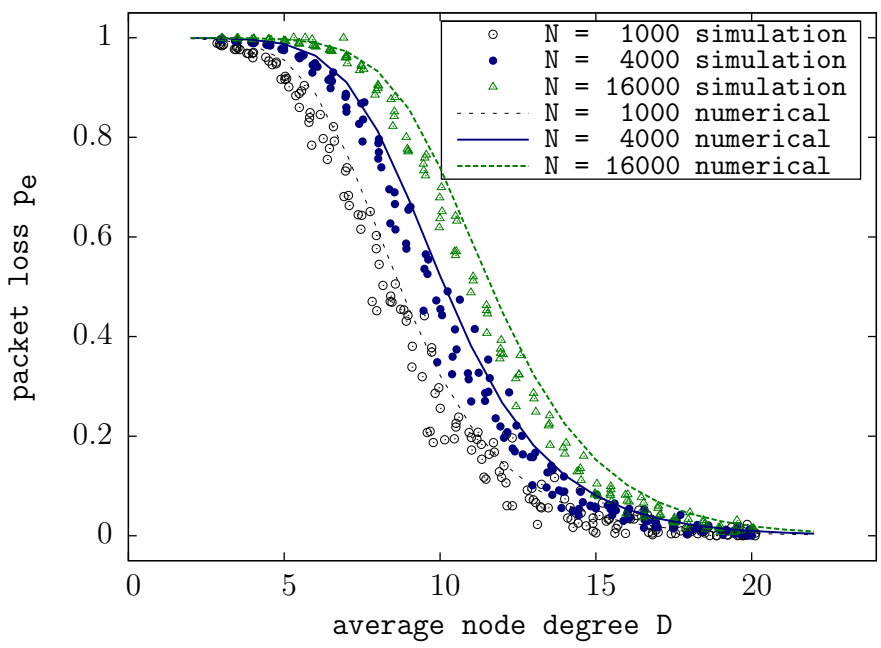

Figure 6. Packet loss probability: numerical computation vs. simulation.

We numerically compute packet loss probability derived above. Figure 6 shows the analytical results compared with the curves fitting simulation data. We can see that the form of the analytical curve corresponds to the curve that fits simulation data, the slight difference coming from several approximations made in the derivation of packet loss probability. To evaluate the approximation error, we can compare the critical average node rank $\bar{k}_{0}=10.15$ obtained from the numerical results for $N=4000$ with the corresponding value of $\bar{k}_{0}=9.90$ given by simulation data.

\section{Conclusion and Future Research}

The goal of this paper is to analyze greedy geographical routing in spontaneous wireless mesh networks. Although we have used a simplistic model based on the unit disk graph, our analysis yields several interesting properties. First, we can approximate the dependence of packet loss probability on the mean node rank with a Fermi-Dirac like function. When the mesh size increases, it becomes more opaque to packets for given average node rank $\bar{k}_{0}$. We also show by simulation that packet loss probability in mesh networks with greedy geographical routing does not exhibit the behavior of a percolating quantity. Finally, we propose an analytical model of greedy geographical routing and use it to derive packet loss probability.

To improve performance of simple greedy forwarding we explore two directions. First, the identification of concave nodes allows fixing the topology defects-one can imagine a process of placing additional mesh routers near concave nodes to limit packet loss. Second, we can enhance greedy routing with an adaptation mechanism to adjust the direction towards the destination if greedy routing forwards packets to concave nodes.

\section{ACKNOWLEDGEMENT}

This work was partially supported by the European Commission project WIP under contract 27402 and the French Ministry of Research project AIRNET under contract ANR05-RNRT-012-01.

\section{REFERENCES}

[1] S. Capkun, M. Hamdi, and J. P. Hubaux, "GPS-Free Positioning in Mobile Ad-hoc Networks," Cluster Computing, vol. 5, no. 2, pp. 157167, April 2002.

[2] P. Bose, P. Morin, I. Stojmenovic, and J. Urrutia, "Routing with Guaranteed Delivery in Ad Hoc Wireless Networks," Wireless Networks, vol. 7, no. 6, pp. 609-616, November 2001.

[3] K. Gabriel and R. Sokal, "A New Statistical Approach to Geographic Variation Analysis," Systematic Zoology, vol. 18, pp. 259-278, 1969

[4] G. Toussaint, "The Relative Neighborhood Graph of a Finite Planar Set," Pattern Recognition, vol. 12, no. 4, pp. 261-268, 1980.

[5] F. Kuhn, R. Wattenhofer, Y. Zhang, and A. Zollinger, "Geometric AdHoc Routing: of Theory and Practice," in Proc. ACM PODC, 2003.

[6] H. Frey, "Scalable Geographic Routing Algorithms for Wireless Ad-Hoc Networks," IEEE Network, July/August 2004.

[7] S. De, A. Caruso, T. Chaira, and S. Chessa, "Bounds on Hop Distance in Greedy Routing Approach in Wireless Ad Hoc Networks," International Journal on Wireless and Mobile Computing, vol. 1, no. 2, pp. 131-140, 2006.

[8] V. Beffara and V. Sidoravicius, "Percolation theory," Encyclopedia of Mathematical Physics, Elsevier 2006.

[9] C. Bettstetter, "On the Minimum Node Degree and Connectivity of a Wireless Multihop Network," in Proc. ACM MOBIHOC, 2002.

[10] I. Glauche, W. Krause, R. Sollacher, and M. Greiner, "Continuum Percolation of Wireless Ad-hoc Communication Networks," Physica: Statistical Mechanics and its Applications, vol. 325, no. 3-4, pp. 57760, 2003.

[11] "Distances in Bounded Regions," http://www.mathpages.com. 